\title{
A European ELT design study
}

\section{Arne Ardeberg, Torben Andersen and Mette Owner-Petersen}

Lund Observatory, Lund University

arne@astro.lu.se, torben.andersen@astro.lu.se, mette@astro.lu.se

\begin{abstract}
For a European ELT, we discuss science requirements and technical options. Science includes from the solar system to the first stars and galaxies. ELT and instrument needs are commented. Design options are discussed for the primary mirror, its segmentation and shape, and an adaptive secondary mirror. Adaptive optics and enclosure are commented.
\end{abstract}

Keywords. ELT, planets, star formation, galaxies, mirrors, adaptive optics, spatial resolution

\section{Why an ELT?}

The rapid advance of astronomy is largely due to the Very Large Telescopes (VLTs), now with adaptive optics (AO). Many problems have been solved and new challenges defined. Diffraction-limited optical to mid-infrared (MIR) imaging with Extremely Large Telescopes (ELTs) opens a new era for spatial and spectral resolution and faint objects. Cutting edge science includes the solar system, formation of stars and planetary discs, stellar evolution, planetary systems, the evolution of galaxies, large scale structure, the first galaxies and ionisation. The regime of the unexpected is vital. Large efforts have gone into the science case (Najita \& Strom 2003, Strom et al. 2003, Gilmozzi 2004, Ardeberg 2004, Hook 2004, Codona 2004), defining the ELT design.

\section{Solar system}

An ELT resolves surfaces and shapes of distant planets, moons and asteroids. For Io and Triton, multi-wavelength time series of subsurface and surface activity, atmospheric ejections and pattern evolution trace life signatures. Space-craft fly-by offers powerful comet snapshots but cannot combine spatial resolution, light collection and time series before, during and after perihelion passage. VLTs offer first Kuiper-Belt Object (KBO) data but an ELT is required for their nature. Binary KBO diameters, light curves and radial velocities can be probed (Snel et al. 2004). Masses, densities and collision data will reveal KBO evolution. KBO shapes and rotations disclose solar-system evolution. For large KBOs, atmospheres can be studied, as an ELT allows faint probe stars.

\section{Formation of stars, circumstellar discs and old stars}

For emerging star-planet systems, AO VLT resolution is too poor. We see star formation initiated by high-mass stars covered by interstellar dust (Nürnberger et al. 2002, Nürnberger \& Petr-Gotzens 2002, Nürnberger \& Stanke 2003, Nürnberger 2003). Accretion of massive stars has been rejected, small-star merging preferred (Bonell et al. 1998), notably in dense regions (Bally \& Zinnecker 2005). This was queried by Yorke \& Sonnhalter (2002). From VLT AO data on large-mass formation, bipolar mass outflows and even larger accretion-disc masses, Chini et al. (2004) concluded that accretion forms also very massive stars. The stellar initial mass function needs further work (Chabrier 


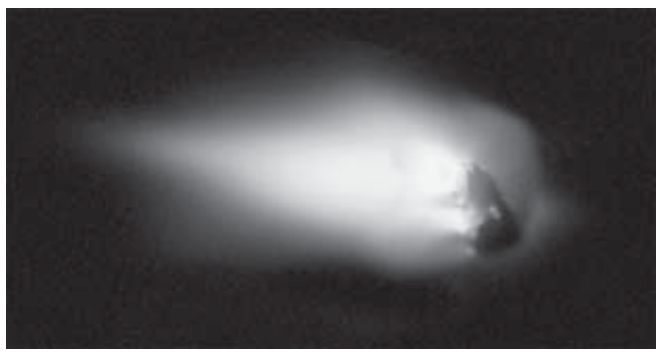

Figure 1. Comet Halley nucleus. Giotto fly-by 1986. Largest nucleus extension is $16 \mathrm{~km}$.

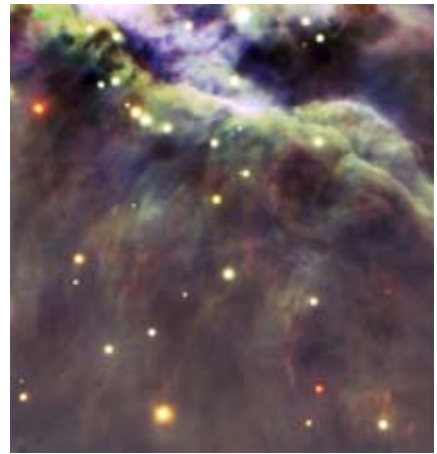

Figure 2. Trapezium star formation (Gemini picture).

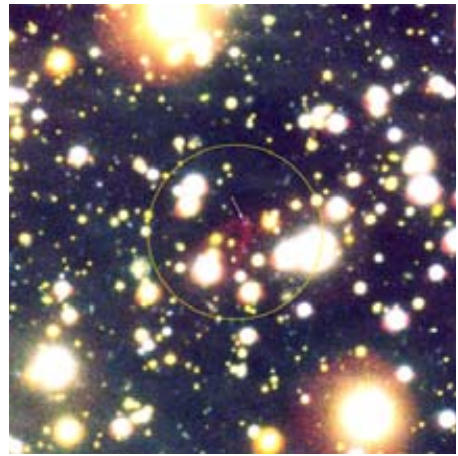

Figure 3. Bowshock nebula near the neutron star RXJ1856.5-3754 (ESO VLT image).

2003). High-contrast ELT images, spectroscopy and dual-linear polarimetry (Vakili 2003) can provide answers, also for smaller stars.

Achernar's flat shape (Domiciano et al. 2003) and apparent stability stresses our weak knowledge of stellar physics. Asteroseismology has proven reliable for solar evolution. For other stars, signal-to-noise ratios are serious limitations (Christensen-Dalsgaard 2005). Real advance requires an ELT. White dwarfs, neutron stars and black holes are faint objects. An ELT will allow millisecond-resolved photometry of micro-variability and evolution of surface features, accretion gas flows and rapid non-radial oscillations and flares.

\section{Planetary systems, exoplanets and signatures of life}

The transformation of circumstellar discs into planets is only vaguely known (Mouillet 2004). The small angular sizes, faintness and power-law mass distribution, the closeness to central stars and high speed of events make studies hard, even with favourable space orientation and distance. Detailed study has been possible in a few cases only. Results indicate a large diversity. Understanding of planet formation requires ELT spatial resolution and light collection. A high frequency of circumstellar discs around forming stars is indicated by $K$-band silhouette images (Elson et al. 2003). Imaging, photometry, polarimetry and spectroscopy are required, with high spatial resolution, for studies of linear sizes, masses, morphology, chemical composition and dynamics.

Direct VLT exoplanet imaging is possible only of high-mass planets orbiting faint stars, emerging stars and brown dwarfs. Imaging and spectroscopy of less massive planets orbiting solar-type stars cannot be achieved without an ELT. While Earth-like planets are a true challenge even with ELTs, they are fully impossible targets for current telescopes. 


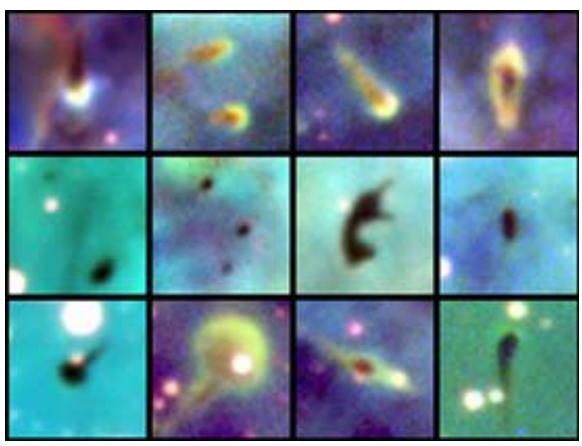

Figure 4. Carina proplyds (Images Nathan Smith, John Bally, Jacob Thiel, Jon Morse U.Colorado/CTIO/NOAO/AURA/NSF).
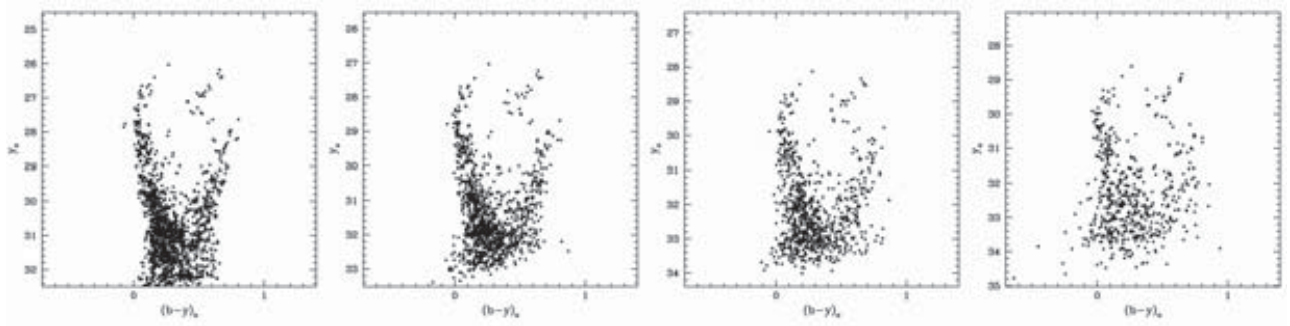

Figure 5. Simulated ELT star cluster CMDs at 4, 8 and 16Mpc (Ardeberg \& Linde 2006).

\section{Evolution of galaxies}

Galaxy evolution and interaction are only vaguely understood. Hierarchical galaxy merging has problems with galaxies 10Gyr old (Cimatti et al. 2004, Glazebrook et al. 2004). Our weak insight includes Local Cluster (LC) galaxies, even the relation between the Galaxy and M31, being both similar (Stephens et al. 2003) and dissimilar (Brown et al. 2003). The LC is not a representative galaxy sample, which the Virgo cluster of galaxies is, however at $16 \mathrm{Mpc}$. Virgo galaxy studies require an ELT.

Field populations in galaxies are hard to study (Ardeberg \& Andersen 2005, Ardeberg \& Linde 2005). Stellar clusters are favourable. An ELT minimises crowding, allowing accurate photometry of cluster stars even beyond the Virgo cluster, detailed colourmagnitude (CMDs) and metallicity diagrams (MDs). High-quality ages can be derived from turn-off-point (TOP) photometry and metallicities from $m_{1}$ versus $(b-y)$ (Ardeberg $\&$ Linde 2006). Integral cluster photometry gives fair ages for very distant galaxies.

\section{Galaxies, large scale structure and expansion}

Large scale structure and cosmic expansion studies have emphasised distance indicators, in the Galaxy (Kubiak \& Udalski 2003, Kervella et al. 2004), the Magellanic Clouds (Soszynski et al. 2003) and far beyond (Freedman et al. 2001, Saha et al. 2001, Sandage et al. 2004, Romaniello et al. 2004). GAIA (Turon et al. 2005) will provide accurate astrometry, parallaxes, multi-band photometry and spectroscopy covering the entire Galaxy, allowing vastly improved distance-reference calibrations for the ELT era. ELT data will advance observations of both local and distant expansion fields and cosmological parameters.

Today, spectroscopic galaxy redshifts reach $z=6-7$, while an ELT will reach $z=10$. The James Webb Space Telescope will identify high- $z$ galaxies. This and new 


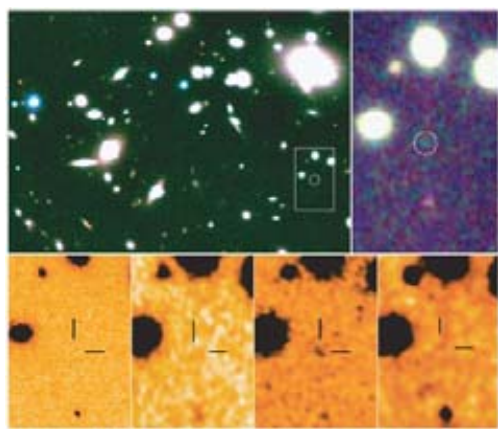

Figure 6. Distant galaxy Abell 1835 IR1916 (from ESO VLT images).

surface-distribution predictions (Bremer et al. 2004, Bremer \& Lehnert 2005) indicate that such galaxies for ELT photometry and spectroscopy should be plentiful.

\section{First galaxies and ionization}

The Wilkinson Microwave Anisotropy Probe (WMAP), Sloan Digital Sky Survey (SDSS) and other studies show the early Universe (Seife 2003, Fan et al. 2004, Richards et al. 2004, Rojas et al. 2005, Springel et al. 2005), from first quantum variations to micro-wave background fluctuations. Consistency requires more data on initial formation and evolution of stars, galaxies, clusters of galaxies, filaments, voids, dark matter, dark energy, ionisation and re-ionisation. The hydrogen had, 380000 years after Big Bang, cooled to a neutral state. Later, it became and remained ionised.

The re-ionisation of the inter-galactic medium (IGM) is poorly understood. While WMAP data point to a partly ionised IGM 500Myr after Big Bang (Kogut et al. 2003, Panagia et al. 2005), early-quasar data show ionisation first at $1 \mathrm{Gyr}$ after Big Bang (Becker et al. 2001). This may indicate two sets of sources of ionisation, a more continuous re-ionisation process or some misinterpretation. We guess that the IGM re-ionised due to UV radiation from pioneer stars and galaxies. However, we do not know how matter inhomogeneity produced the first galaxies and stars nor how ionisation of galaxies led to a completely ionised IGM. VLTs can give a first picture of the star formation of galaxies back to $1 \mathrm{Gyr}$ after Big Bang. In any picture, then the IGM was re-ionised. Adequate studies require galaxy data at 0.5 to $1.0 \mathrm{Gyr}$ after Big Bang. We need optical-visual and NIR ELT data, imaging, photometry and identification spectroscopy.

\section{The unexpected}

Each new telescope, notably larger than its predecessors, has exceeded science expectations. Still, its greatest contributions have been those impossible to predict. With its leap in size and image quality, an ELT will reveal unexpected processes and dimensions.

\section{AO, photometry and polarimetry}

Much ELT science needs photometry, shorter wavelengths emphasised. Most CMD work can do with a precision of $0.03-0.06 \mathrm{mag}$, but MDs require $0.01-0.03 \mathrm{mag}$. AO photometry stable in time and field is a challenge. We need end-to-end modelling and optical path differences (OPDs) converted to point-spread functions (PSFs), in progress 
in Lund. ELT AO will allow wavelengths larger than 1000-2000nm at first light, opticalvisual data a few years later, important for crowded-field photometry.

Many programmes need (spectro-)polarimetry. Star formation and circumstellar discs are examples as are stars distorted by non-radial pulsations and rotation. Other examples are brown dwarfs, jets of active galactic nuclei and the interstellar medium.

\section{ELT science case, instrumentation and interaction}

Science requires wavelengths from 350 to at least $20000 \mathrm{~nm}$. The spatial resolution needed is around 10 mas at $2000 \mathrm{~nm}, 2-3$ mas in the optical-visual, the spectral resolution from $R=5$ to $R=10^{5}$ and beyond. Many programmes can work with a 1 arcsec FoV, other need 5-10 arcmin. Single-conjugate AO (SCAO) covers FoVs of 1 arcmin, multiconjugate AO (MCAO) of 2 arcmin, ground-layer AO (GLAO) larger fields with modest PSF. Earth-like planet studies require contrast ratios of and beyond $10^{10}$, while $10^{7}$ is enough in most other cases. Other requirements are, except solid photometry, rapid access, time resolution, time series, long exposures, astrometry and coronagraphy.

Even a sophisticated ELT is of little use if not equipped with auxiliary instruments of similar class. High-quality interfacing of the ELT and its instrumentation is essential. The design must include gravity invariant and spacious instrument platforms. Any ELT instrument concept must be developed, taking science requirements into account.

So far, science case and ELT development have progressed mainly in parallel. When ELT development gets more intensive, improved rapports are needed. A telescope simulation engine should produce PSFs, including time and position variations. End-to-end modelling should involve science, telescope and instrument teams.

\section{Primary mirror options}

Establishing a 50m reflecting primary mirror (M1) is a challenge. Some basic options are available related to potential segmentation. The choice has considerable bearing not only on M1 but also on the telescope structure and the AO system.

Conceptually, a large number of small rigid segments with an edge-to-edge size of $15 \mathrm{~cm}$ may be envisaged. Such a mirror could also serve as an adaptive mirror for correction of telescope and atmospheric aberrations. $10^{5} \mathrm{M} 1$ segments will be needed for a $50 \mathrm{~m}$ ELT, implying very complicated phasing problems. If M1 segments of 1-2m size are chosen, the number of segments decreases to 600-2400 for a 50m ELT. All segments need tip/tilt and piston control with feedback from edge sensors and wavefront sensors. The concept is proven for the Keck telescopes, and the number of segments is well within handling limits. This is seen as a safe concept.

Another option is to choose fully flexible segments somewhat larger than $8 \mathrm{~m}$. For a 50m ELT, 30-40 segments are required. This concept is basically attractive but unproven. Also, the long, soft segment edges imply phasing problems. This solution may be the option for a generation of telescopes succeeding the ELTs.

At least in principle, the most attractive concept is that of a fully monolithic M1 offering full freedom from phasing problems but implying significant fabrication difficulty. On-site fabrication will be a necessity. Although a monolithic M1 may be an attractive challenge for the future it is not a realistic option at this time of development.

In conclusion, a primary mirror made up of rigid segments in the $1-2 \mathrm{~m}$ range seems to be most attractive at the moment. 


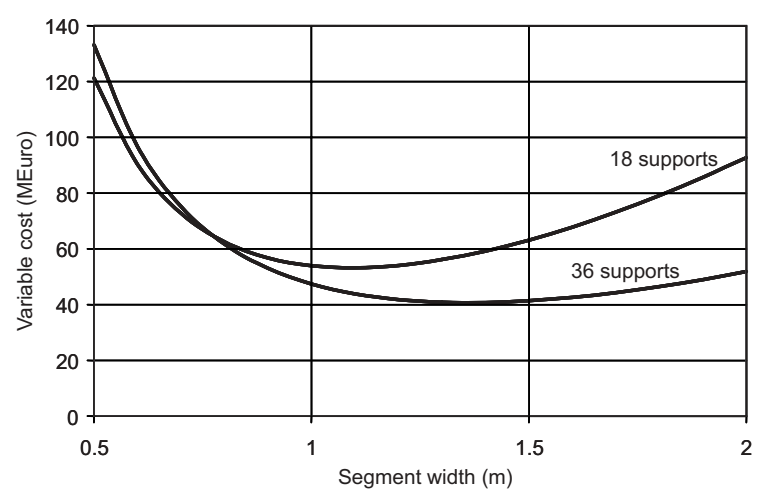

Figure 7. Estimate of part of the cost of M1 that is variable versus segment edge-to edge size. Cost is in MEuros and segment size in meter.

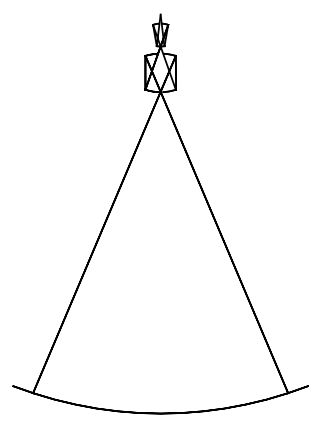

A

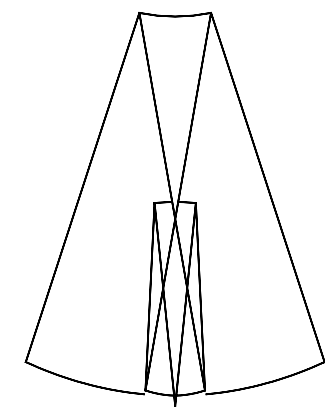

B

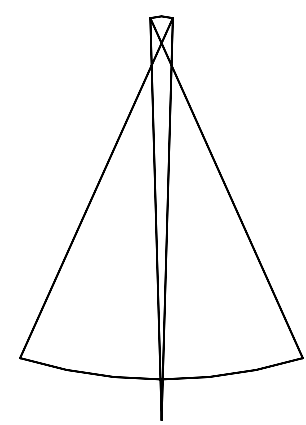

C

Figure 8. ELT M1 options. A: Hobby-Eberly, B: Meinel, C: Gregorian.

\section{Size and cost of M1 segments}

With fabrication, handling and phasing considerations pointing to M1 segments of 1$2 \mathrm{~m}$, it is essential to consider the relation between segment size and cost for selection of optimum segment size. A cost estimate must include not only the segment blanks and figuring but also those of mirror supports and structure, actuators and edge sensors. Corresponding calculations have been made. Figure 7 reports the part of the cost of M1 that is variable versus segment edge-to edge size. Included are relevant costs of supports, actuators, mirror blanks, structure and edge sensors. We conclude that between 1 and $2 \mathrm{~m}$, the curves are rather flat and the choice is quite open.

\section{Form of primary mirror}

In the discussion of ELT concepts, both aspherical and spherical shapes of M1 have been advocated, with the former dominating. The choice between these options has a large influence on the total opto-mechanical design of the ELT. For both concepts, different optical layouts exist. Figure 8 illustrates this.

The Arecibo concept combining a spherical M1 with a compact corrector design is a well-known option. This concept was chosen for the Hobby-Eberly Telescope (HET, see A of Fig. 8) and the South Africa Large Telescope (SALT). It was also the choice 


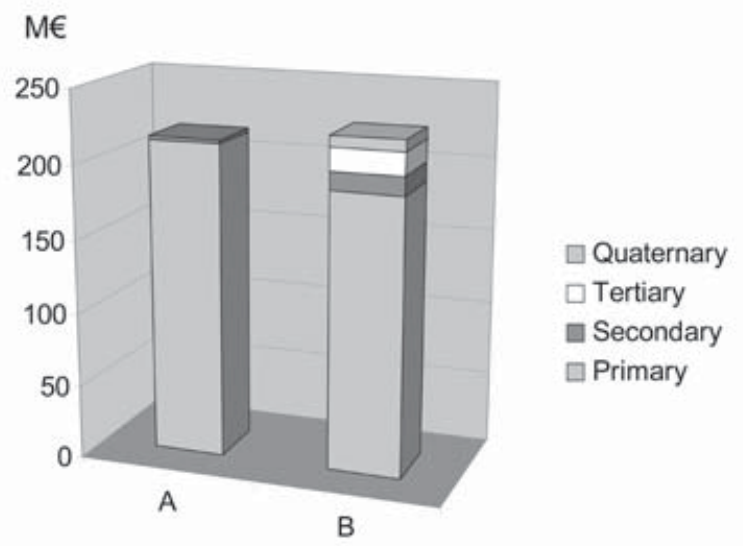

Figure 9. Comparison of cost of blanks and figuring of all mirrors between two 50m ELTs, one of Meinel-type with a spherical M1, the other the Euro50.

for ESOs OverWhelmingly Large Telescope (OWL) concept with the addition of a flat secondary mirror (M2).

Another option for an ELT with a spherical M1 is the four-mirror configuration suggested by Meinel \& Meinel (1992). The three reflecting surfaces following M1 are distributed in a configuration less compact than for the Arecibo concept. An alternative configuration is the folded spherical telescope concept proposed by Wilson et al. (1994).

If an aspherical M1 is chosen, two main configurations seem attractive, the Cassegrain and the Gregorian. The Cassegrain solution is more compact but does not favour an adaptive M2 because of the difficulty of measuring the form of a convex mirror in-situ. The Gregorian solution is excellent for an adaptive M2 because of its concave form, at the price of a somewhat longer telescope. A further advantage of the Gregorian solution is that the secondary is conjugate to an atmospheric layer near ground.

A cost comparison between the conceptual alternatives should take the ELT aperture into account, as the mass-production savings on identical spherical segments is a function of the surface of M1. However, it is equally important to include the cost of all mirrors, as highly aspheric mirrors may be cost drivers even for moderate sizes.

Through contacts with optical manufacturers, we have attempted a cost comparison between two 50m ELTs, one of the Meinel type, the other the Euro50 of Gregorian type. Including the cost of blanks and figuring of all mirrors, four in the Meinel case, two for Euro50, we obtained the results shown in Fig. 9. We conclude that an aspherical M1 has a cost advantage over a spherical M1. However, the difference is not large, and considering the cost of a complete ELT, the difference seems negligible.

For an ELT with an aperture considerably larger than $50 \mathrm{~m}$, the result may well be different. Still, however, we caution concerning the complications caused by several reflecting surfaces, for instance related to light loss, stray light, IR performance, internal polarisation, mechanical stability tolerances and AO configuration difficulties.

\section{Position of AO}

The deformable mirrors of an AO system can be located at different positions. These are M1, M2, the tertiary, quaternary and following mirrors as well as post-focus including relay optics. While a single conjugate $\mathrm{AO}$ telescope can be built with a total of two mirrors only, multi-conjugate (and even dual-conjugate) AO requires at least five mirrors. 

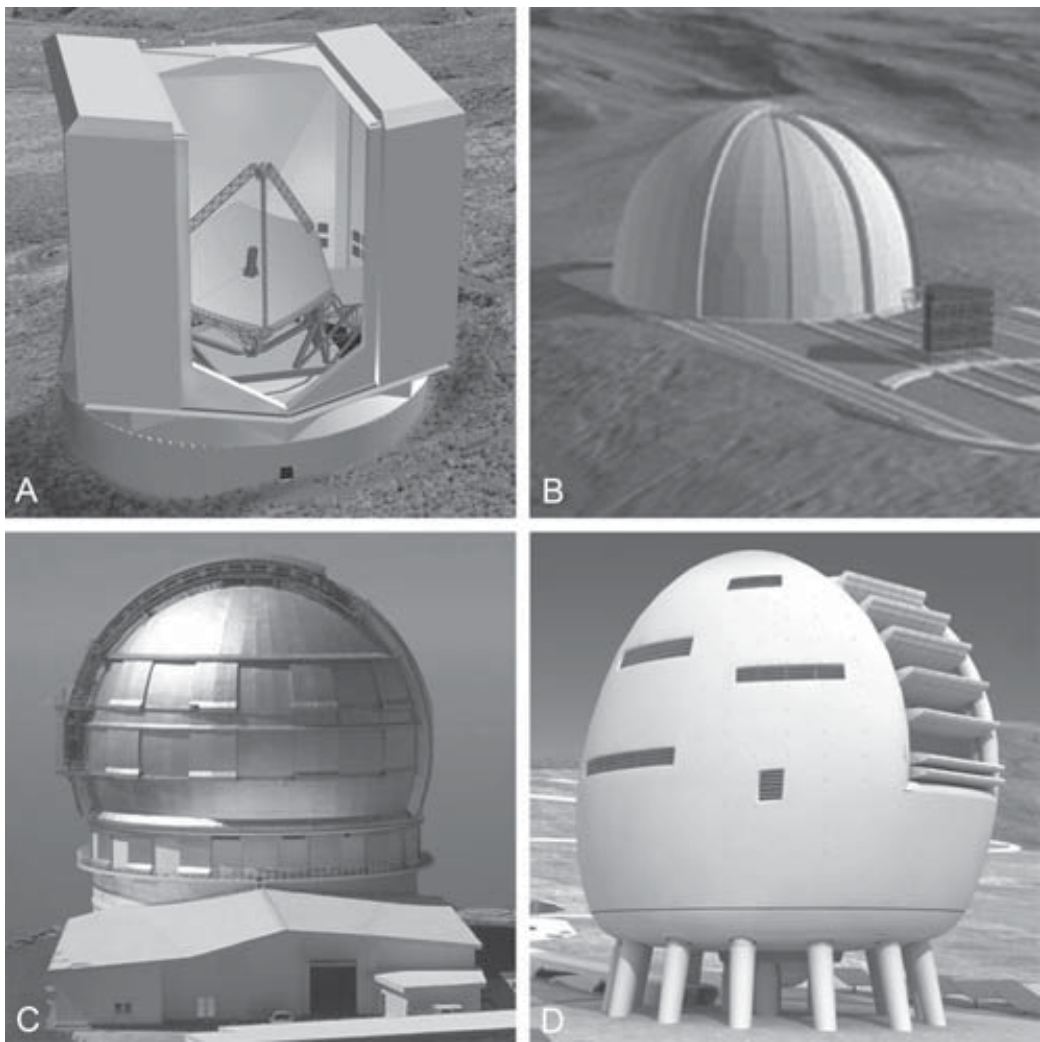

Figure 10. Four ELT enclosure concepts. A: Box (Euro50), B: Shelter (OWL, courtesy ESO), C: Dome (GTC, courtesy Grantecan), D: Flow-optimized shell (courtesy E-BAT consortium, Sweden).

For a two-mirror ELT with pre-focus AO, development of adaptive 2-4m secondary mirrors is an urgent requirement. Reduction of cost in comparison with the existing large deformable mirrors is important, for instance through replication, or new actuator or sensor designs. A large dynamic range is a necessity for the deformable mirrors because wind loads introduce wavefront aberrations up to $20 \mu \mathrm{m}$. For the Euro50, the $4 \mathrm{~m}$ deformable secondary mirror has well over 1000 eigenmodes within the servo passband of around $1 \mathrm{kHz}$. For a wavelength of $2 \mu \mathrm{m}$ more than 10000 actuators are required.

\section{Enclosure options}

With the size of an ELT, the choice of enclosure configuration is important both from a cost and operation point-of-view. For cost reasons, it is doubtful whether more traditional enclosure designs are possible for ELTs with apertures over $60 \mathrm{~m}$. There are three major design options, domes, "boxes" and moving shelters. In all cases, active air flushing and close temperature control inside the enclosure are essential features. In Fig. 10, four enclosure concepts are illustrated.

The enclosure for a $50 \mathrm{~m}$ telescope will be among the largest buildings in the world, and its cost is a significant part of the total ELT budget. Hence, it is essential to keep it as low as possible. We have calculated the cost of a box enclosure as a function of M1 size for an ELT and the relationship between enclosure cost and primary mirror diameter is nearly parabolic. The results are given in Fig. 11 . 
Figure 11. Cost of ELT enclosure in MEuros versus M1 diameter in meter. Relation valid for a box construction.

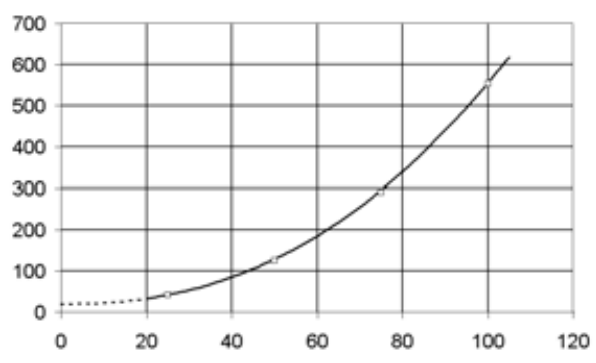

\section{Systems design}

The most significant challenge for design and construction of an ELT is related to wavefront control, i.e. to design of passive support structures and automatic control systems that correctly maintain form and position of the optical elements. The telescope is subjected to gravity and wind forces that tend to distort the structure statically and dynamically. In addition, the atmosphere will deteriorate image quality. Adaptive optics will correct not only for atmospheric aberrations but together with numerous other automatic control systems will correct for aberrations from wind, gravity, atmosphere and thermal loads. Prediction of the performance of an ELT and its automatic control systems is of utmost importance and one of the most significant challenges for ELT design. The most important tool for this is integrated modelling, i.e. computer simulations including effects from optics, structure and control systems.

\section{A concerted European effort}

At the present symposium, presentations have been made of four ELT concepts, regarded as mature (Carlberg 2006). Two of them have been developed in North America, two in Europe. The work on the Thirty-Meter Telescope (TMT) is performed by a USCanadian team and based on earlier studies of the California Extremely Large Telescope (CELT), the Giant Segmented Mirror Telescope (GSMT) and the Very Large Optical Telescope (VLOT). The development of the Giant Magellan Telescope (GMT), with an equivalent aperture of $21.4 \mathrm{~m}$, is undertaken by a team supported by eight US institutions. In Europe, the OWL has been developed by a European Southern Observatory (ESO) group of experts, while the Euro50 is supported by five European institutes and co-ordinated by a group at Lund Observatory.

Both the TMT and the GMT have their concepts well defined and development work advances well. The GMT team has already, highly successfully, produced one of the seven M1 mirror blanks. Both American ELT projects are highly interesting and promising and may be realized and commissioned within around 10 years.

The European Commission (EC) of the European Union (EU) takes, via OPTICON, a European co-ordination network for astronomy in the optical-visual and NIR wavelength bands, active part in the work aimed at a European ELT. The EC has explicitly asked for a common European ELT concept. It has also, under its Frame Programme 6 (FP6) allocated 8 MEuros for the development of such a concept. Work on a variety of ELT items is also well under way.

Further progress of the European ELT requires, at some time, a clear telescope concept. We feel that the European community is now ready to decide on a final overall telescope design and propose immediate action to define a common European ELT concept. While both OPTICON and ESO have major roles to play in this context, the Euro50 team will be happy to offer its full support. 


\section{References}

Ardeberg, A. 2004, SPIE 5489, 23

Ardeberg, A. \& Andersen, T. 2005, Proc. JEMAM Granada 2004, in press

Ardeberg, A. \& Linde, P. 2005, Proc. JENAM Granada 2004, in press

Ardeberg, A. \& Linde, P. 2006, this volume

Bally, J. \& Zinnecker, H. 2005, AJ 129, 2281

Becker, R.H., Fan, X., White, R.L. \& Strauss, M.A. et al. 2001, AJ 122, 2850

Bonell, I., Bate, M. \& Zinnecker, H. 1998, MNRAS 298, 93

Bremer, M.N., Lehnert, M.D., Waddington, I. et al. 2004, MNRAS 347, L7

Bremer, M. \& Lehnert, M. 2005, Proc. ESO Symp. "Exploring the Cosmic Frontier" in press

Brown, T.M., Fergusson, H.C., Smith, E., Kimble, R.A. et al. 2003, ApJ 592, L17

Carlberg, R. 2006, this volume

Chabrier, G. 2003, PASP 115, 763

Chini, R., Hoffmeister, V., Kimeswenger, S., Nielbock et al. 2004, Nature 429, 155

Christensen-Dalsgaard, J. 2005, Proc. ESA SP-560, 13th Cool Stars Workshop, 81

Cimatti, A., Daddi, E., Renzini, A., Cassata, P., Vanzella, E. et al. 2004, Nature 429, 155

Codona, J.L. 2004, SPIE 5490, 379

Domiciano de Souza, A., Kervella, P., Jankov, S., Abe, L. et al. 2003, A\&A 407, L47

Elston, R.J., Lada, E.A., Alves, J., Ferreira, B., Lada, C.J. et al. 2003, AAS 202, 2809

Fan, X., Hennawi, J.F., Richards, G.T., Strauss, M.A. et al. 2004, AJ 128, 515

Freedman, W.L., Madore, B.F., Gibson, B.K., Ferrarese, L. et al. 2001, ApJ 553, 47

Gilmozzi, R. 2004, SPIE 5489, 1

Glazebrook, K., Abraham, R.G., McCarthy, P.J. et al. 2004, Nature 430, 181

Hook, I. 2004, SPIE 5489, 35

Kervella, P., Bersier, D., Mourard, D., Nardetto, N., Fouqué, P. et al. 2004, A\&̇A 428, 587

Kogut, A., Spergel, D.N., Barnes, C. et al. 2003, ApJS 148, 161

Kubiak, M. \& Udalski, A. 2003, Acta Astron. 53, 117

Meinel, A.B. \& Meinel, M.P. 1992, Opt. Eng. 31, 2271

Mouillet, D. 2004, Science 303, 1982

Najita, J. \& Strom, S.E. 2003, SPIE 4835, 1

Nürnberger, D.E.A. 2003, A\& A 404, 255

Nürnberger, D.E.A., Bronfman, L., Yorke, H.W. \& Zinnecker, H. 2002, A\&SA 394, 253

Nürnberger, D.E.A. \& Petr-Gotzens, M.G. 2002, A\& $A$ 382, 537

Nürnberger, D.E.A. \& Stanke, Th. 2003, A\&SA 400, 223

Panagia, N., Fall, S.M., Mobasher, B. et al. 2005, ApJ 633, L1

Richards, G.T., Nichol, R.C., Gray, A.G. et al. 2004, ApJS 155, 257

Rojas, R.R., Vogeley, M.S., Hoyle, F. \& Brinkmann, J. 2005, ApJ 624, 571

Romaniello, M., Primas, F., Mottini, M. \& Groenewegen, M. 2004, IAU Coll. 193, 426

Saha, A., Sandage, A., Thim, F. \& Labhardt, L. et al. 2001, ApJ 551, 973

Sandage, A., Tammann, G.A. \& Reindl, B. 2004, A\& $A$ 424, 43

Seife, Ch. 2003, Science 302, 2038

Snel, R., Ardeberg, A. \& Flicker, R. 2004, SPIE 5382, 57

Soszynski, I., Udalski, A., Szymanski, M., Kubiak, M. et al. 2003, Acta Astron. 53, 93

Springel, V., White, S.D.M., Jenkins, A., Frenk, C.S. et al. 2005, Nature 435, 629

Stephens, A.W., Frogel, J.A., DePoy, D.L., Freedman, W. et al. 2003, AJ 125, 2473

Strom, S.E., Stepp, L.M. \& Gregory, B. 2003, SPIE 4840, 116

Turon, C., O'Flaherty, K.S. \& Perryman, M.A.C. (eds.) 2005, ESA SP-576

Vakili, F. 2003, in: C. Aime \& R. Soumner (eds.), Astronomy with High Contrast Imaging, EAS Publ. Ser. 8, 49

Yorke, H. \& Sonnhalter, C. 2002, ApJ 569, 846

Wilson, R.N., Delabre, B. \& Franza, F. 1994, SPIE 2199, 1052. 


\section{Discussion}

DENNEFELD: We heard from GMT and TMT presentations that reasonable extrapolations from present large telescopes were a fundamental point in their design. What was/is the driver for your choice of $50 \mathrm{~m}$, which was proposed already long ago?

Ardeberg: Our first design was of a 25m telescope. Then, after 1996, we were rapidly convinced that adaptive optics was a realistic option for future telescope design. Given the first generation observations of exoplanetary systems and accepting the challenge of an AO ELT, we optimized our design efforts for a $50 \mathrm{~m}$ aperture. We still feel that a $50 \mathrm{~m}$ aperture ELT is a highly challenging yet realistic option. 